\title{
Diabetes, bilirubin and amputations: is there a link?
}

\author{
N. Katsiki • A. Karagiannis • D. P. Mikhailidis
}

Received: 4 January 2013 / Accepted: 14 January 2013 / Published online: 27 January 2013

(C) Springer-Verlag Berlin Heidelberg 2013

\begin{abstract}
A study by Chan et al in this issue of Diabetologia (DOI:10.1007/s00125-012-2818-4) reports that low plasma bilirubin levels are associated with an increased risk of amputation in patients with type 2 diabetes mellitus participating in the Fenofibrate Intervention and Event Lowering in Diabetes (FIELD) study. These findings raise the interesting and clinically relevant hypothesis that bilirubin protects against risk of amputation in patients with type 2 diabetes. This commentary considers some of the limitations associated with research aiming to define any link between circulating bilirubin levels and vascular disease. Numerous confounding factors (several of which may be present in patients with type 2 diabetes) may explain why the literature regarding this potentially protective role of bilirubin remains controversial.
\end{abstract}

Keywords Amputation · Bilirubin - Diabetes mellitus · Fenofibrate $\cdot$ Smoking $\cdot$ Vascular disease

$\begin{array}{ll}\text { Abbreviations } \\ \text { FIELD } & \begin{array}{l}\text { Fenofibrate Intervention and Event Lowering in } \\ \text { Diabetes }\end{array} \\ \text { NAFLD } & \begin{array}{l}\text { Non-alcoholic fatty liver disease } \\ \text { PAD }\end{array} \\ \text { Peripheral artery disease }\end{array}$

In this issue of Diabetologia, Chan et al [1] report the results of a pre-specified subsidiary analysis of the Fenofibrate Intervention and Event Lowering in Diabetes (FIELD)

\footnotetext{
N. Katsiki $\cdot$ A. Karagiannis

Second Propedeutic Department of Internal Medicine,

Medical School, Aristotle University of Thessaloniki,

Hippokration Hospital, Thessaloniki, Greece

D. P. Mikhailidis $(\bowtie)$

Department of Clinical Biochemistry (Vascular Disease Prevention Clinic), Royal Free Hospital campus, University College London Medical School, University College London (UCL), Pond Street, London NW3 2QG, UK

e-mail: mikhailidis@aol.com
}

study. This trial included 9,795 patients with type 2 diabetes mellitus. Lower bilirubin levels increased the risk of first amputation (HR 1.38 per $5 \mu \mathrm{mol} / \mathrm{l}$ decrease in bilirubin concentration; 95\% CI 1.05, 1.81; $p=0.019$ ) after multiple adjustments. These adjustments included age, height, smoking status, $\gamma$-glutamyltransferase activity, $\mathrm{HbA}_{1 \mathrm{c}}$ level and trial treatment allocation (placebo vs fenofibrate), as well as previous peripheral artery disease (PAD), non-PAD cardiovascular disease, amputation or diabetic skin ulcer, neuropathy, nephropathy and diabetic retinopathy. The authors conclude that their findings raise the hypothesis that bilirubin protects against the need for amputation in patients with type 2 diabetes.

The hypothesis for this subsidiary analysis was that several actions attributed to bilirubin that may protect from atherosclerosis (e.g. antioxidant and anti-inflammatory effects) $[1,2]$ might also extend to decreasing the risk of amputation in patients with type 2 diabetes. These findings are not only of interest in terms of explaining the pathogenesis leading to amputation but also in pointing towards future treatment options. There is also a need for useful biomarkers that both predict the risk of amputation and alter outcomes. This would not only improve quality of life but also reduce the considerable cost associated with amputation, especially in patients at high risk of needing this procedure (e.g. those with type 2 diabetes) [1].

The authors discuss several limitations of their study [1]. There were only 190 amputations (115 patients) over the 5 year duration of the FIELD trial [1]. This limits the power of multiple adjustments in specific patient subgroups. Some of these limitations need to be viewed in the context of variables that can influence bilirubin levels. For example, women and smokers have lower bilirubin levels than men and non-smokers, respectively, as was appreciated by the authors [1]. In turn, smokers had a greater risk of amputation than non-smokers, and women had a lower risk than men [1]. Furthermore, there is also an effect of glycaemia on bilirubin levels [1]; for example, others have reported that 
higher $\mathrm{HbA}_{1 \mathrm{c}}$ levels were associated with lower bilirubin levels in patients with type 2 diabetes [3]. This association is of interest because the amputation group had higher $\mathrm{HbA}_{1 \mathrm{c}}$ levels than those who did not need an amputation during the FIELD trial [1]. The amputation group also had a higher mean plasma creatinine level [1]. In this context, we need to consider that fenofibrate can raise plasma creatinine levels [4] and that this fibrate reduced the amputation rate in the FIELD trial (HR 0.64, 95\% CI 0.44, 0.94 after adjusting for bilirubin; $p=0.02$ ) [1].

All the patients in the FIELD trial had type 2 diabetes; therefore, it is of interest that a high total bilirubin level may protect against diabetes (an effect possibly related to actions of bilirubin on glucose metabolism, among other mechanisms) [5-7]. This protection may extend to diabetic nephropathy [6] and retinopathy [8], although the literature is not consistent. Does this mean that, in terms of bilirubin levels, there was an element of unintentional pre-selection in the FIELD trial?

Whatever the limitations of this pre-specified subsidiary analysis of the FIELD study, it is relevant that others have reported a similar link between amputation risk and bilirubin levels in different populations [1].

The use of statins was associated with lower bilirubin levels in one study that included patients at high cardiovascular risk (diabetes, hypercholesterolaemia or vascular disease) [9]. We have shown, in a small study, that bilirubin levels were lower in untreated hypertensive individuals compared with normotensive people or treated hypertensive patients. We attributed this effect to the antioxidant actions of certain drugs used to treat hypertension and to a higher level of oxidative stress in untreated hypertensive patients [10]. These studies suggest that commonly used drug treatments (i.e. for dyslipidaemia and hypertension) may influence bilirubin levels. Lower serum bilirubin levels were also reported in patients with the metabolic syndrome, in which bilirubin negatively correlated with circulating insulin levels and visceral adipose tissue [11]. In a large prospective study (5,900 Korean men; followed up between 2002 and 2009) higher serum direct (conjugated) bilirubin levels were significantly associated with a lower risk of developing nonalcoholic fatty liver disease (NAFLD) [12]. Less than $1 \%$ of the participants in the study had diabetes. One of the limitations of this study [12] is that the diagnosis of NAFLD was based on ultrasound findings instead of the 'gold standard' liver biopsy. However, it is difficult to see how such a large study could cope with the logistics of liver biopsies let alone the ethical restrictions associated with such a procedure. The relevance of these findings is that NAFLD is both common in patients with type 2 diabetes and has been proposed as a predictor of increased vascular risk [13, 14]. However, we were unable to find any literature on NAFLD and risk of amputation. We therefore need to consider whether such effects are relevant to the design of future studies aimed at defining any link between vascular pathology and circulating bilirubin levels.

Despite limitations, the findings of this FIELD prespecified subsidiary analysis [1] are of interest as they extend our knowledge of the link between bilirubin and vascular disease. However, because of the variability in the evidence regarding the role of bilirubin in vascular disease [1, 2], more research is urgently needed in this field. One rapid way of dealing with this problem is to analyse the role of bilirubin in published trials where this information is available [2]. This is a credible option since bilirubin levels may predict risk even in patients taking statins [15]. It goes without saying that future designs of vascular trials should incorporate an analysis of the role of bilirubin.

There may be several reasons for the lack of agreement in the literature regarding how bilirubin might influence vascular pathology. Among these is that different populations (even within a single study) were sampled, and the fact that there are numerous relationships between bilirubin levels and other predictors of vascular risk (including NAFLD, sex and smoking status, as well as fasting insulin, triacylglycerol, $\mathrm{HbA}_{1 \mathrm{c}}$ and exercise levels) $[1,2,16,17]$. Furthermore, bilirubin may reflect other changes that are relevant to vascular risk $[2,18]$. Undoubtedly it is difficult to adjust correctly for all these variables and thus defining the exact role of bilirubin in terms of vascular risk remains elusive.

Duality of interest This commentary was written independently; no company or institution supported the authors financially or by providing a professional writer. NK has given talks and attended conferences sponsored by MSD, Pfizer and Astra Zeneca. AK has given talks and attended conferences and participated in trials sponsored by Novartis, Pfizer and Astra Zeneca. DPM has given talks, attended conferences and participated in trials and advisory boards sponsored by MSD, Genzyme and Abbott.

Contribution statement All authors were responsible for the conception, design and drafting of the manuscript, and approved the final version for publication.

\section{References}

1. Chan KH, O'Connell R, Sullivan DR et al (2013) Plasma total bilirubin levels predict amputation events in type 2 diabetes mellitus: the Fenofibrate Intervention and Event Lowering in Diabetes (FIELD) study. Diabetologia. doi:10.1007/s00125-012-2818-4

2. Breimer LH, Mikhailidis DP (2011) Is bilirubin a marker of vascular disease and/or cancer and is it a potential therapeutic target? Curr Pharm Des 17:3644-3655 
3. Choi SW, Lee YH, Kweon SS et al (2012) Association between total bilirubin and hemoglobin A1c in Korean type 2 diabetic patients. J Kor Med Sci 27:1196-1201

4. Ncube V, Starkey B, Wang T (2012) Effect of fenofibrate treatment for hyperlipidaemia on serum creatinine and cystatin C. Ann Clin Biochem 49(pt 5):491-493

5. Cheriyath P, Gorrepati VS, Peters I et al (2010) High total bilirubin as a protective factor for diabetes mellitus: an analysis of NHANES data from 1999-2006. J Clin Med Res 2:201-206

6. Han SS, Na KY, Chae DW, Kim YS, Kim S, Chin HJ (2010) High serum bilirubin is associated with the reduced risk of diabetes mellitus and diabetic nephropathy. Tohuku J Exp Med 221:133-140

7. Ohnaka K, Kono S, Inoguchi T et al (2010) Inverse associations of serum bilirubin with high sensitivity C-reactive protein, glycated hemoglobin, and prevalence of type 2 diabetes in middle-aged and elderly Japanese men and women. Diabetes Res Clin Pract 88:103-110

8. Yasuda M, Kiyohara Y, Wang JJ et al (2011) High serum bilirubin levels and diabetic retinopathy: the Hisayama Study. Ophthalmology $118: 1423-1428$

9. Ong KL, Wu BJ, Cheung BM, Barter PJ, Rye KA (2011) Association of lower total bilirubin level with statin usage: the United States National Health and Nutrition Examination Survey 19992008. Atherosclerosis 219:728-733

10. Papadakis JA, Ganotakis ES, Jagroop IA, Mikhailidis DP, Winder AF (1999) Effect of hypertension and its treatment on lipid, lipoprotein (a), fibrinogen, and bilirubin levels in patients referred for dyslipidemia. Am J Hypertens 12:673-681
11. Torgerson JS, Lindroos AK, Sjöström CD, Olsson R, Lissner L, Sjöström L (1997) Are elevated aminotransferases and decreased bilirubin additional characteristics of the metabolic syndrome? Obes Res 5:105-114

12. Chang Y, Ryu S, Zhang Y et al (2012) A cohort study of serum bilirubin levels and incident non-alcoholic fatty liver disease in middle aged Korean workers. PLoS One 7:e37241

13. Bhatia LS, Curzen NP, Byrne CD (2012) Nonalcoholic fatty liver disease and vascular risk. Curr Opin Cardiol 27:420-428

14. Athyros VG, Tziomalos K, Gossios TD et al (2010) Safety and efficacy of long-term statin treatment for cardiovascular events in patients with coronary heart disease and abnormal liver tests in the Greek Atorvastatin and Coronary Heart Disease Evaluation (GREACE) Study: a post-hoc analysis. Lancet 376:1916-1922

15. Horsfall LJ, Nazareth I, Petersen I (2012) Cardiovascular events as a function of serum bilirubin levels in a large, statin-treated cohort. Circulation 126:2556-2564

16. Ko GT, Chan JC, Woo J et al (1996) Serum bilirubin and cardiovascular risk factors in a Chinese population. J Cardiovasc Risk 3:459-463

17. Swift DL, Johannsen NM, Earnest CP, Blair SN, Church TS (2012) Effect of different doses of aerobic exercise training on total bilirubin levels. Med Sci Sports Exerc 44:569-574

18. Breimer LH, Mikhailidis DP (2010) Could carbon monoxide and bilirubin be friends as well as foes of the body? Scand J Clin Lab Invest 70:1-5 